\title{
Non-Markovian dynamics of a three-level atom in a stochastic field with finite correlation time due to initial correlations
}

\author{
Victor Mikhailov* and Nikolay Troshkin \\ Department of Physics, Samara National Research University, 443086 Samara, Russia
}

\begin{abstract}
Dynamics of a three-level atom interacting with a stochastically fluctuating environment characterized by finite correlation time is investigated in the cases of a product initial state and a correlated initial state. The correlated initial state is obtained by the state reduction caused by the von Neumann measurement on the quantum system evolved from the product initial state and surrounded by the initially stationary stochastic environment. It is shown that in case of a pure dephasing process under the influence of a Kubo-Anderson type stochastic environment, the projective measurement causes the emergence of correlations between previously independent stochastic processes, their non-stationarity, and also causes the transition from a Markovian-type evolution to a non-Markovian one.
\end{abstract}

Time evolution of a quantum system essentially depends on the character of interaction with a surrounding environment. In case of a weak interaction and small correlation times, dynamics of an open system can be described in the Markovian approximation by a master equation in Lindblad form [1,2]. Any two states of an open system tend to decrease their distinguishability under a Markovian process. The distinctive feature of a non-Markovian dynamics is existence of time intervals where the memory effects induce the growth of distinguishability of quantum states [3,4].

We study the dynamics of a three-level quantum system (a three-level atom) in an external classical stochastic field. The stochastic field is defined by independent stochastic Markovian processes with finite correlation time. The probability density function of each stochastic process satisfies a master equation of the Fokker-Planck type with Gaussian stationary solution. The influence of the stochastic field on the atom induces shifts of its energy levels and spontaneous transitions between them [5].

At the initial moment of time, the quantum system and the stochastic field are in an uncorrelated product state and the stochastic processes relating to the stochastic field are stationary. Then the total system evolves from the product state to some joint state at the moment when the von Neumann measurement is performed [6]. After the measurement, the wave function of the atom subsystem collapses, and its further evolution continues from a given pure state. The stochastic field state also undergoes significant alteration as a result of the measurement.

\footnotetext{
*Corresponding author: va_mikhailov@mail.ru
} 
To find the stochastic field state after the measurement, we consider the joint evolution of the atom and the stochastic processes, which can be described by the stochastic Liouville's equation [7-9]. In general, solving the stochastic Liouville's equation is complicated by its complex superoperator structure. For example, it could be solved by the method of hierarchical equations of motion (HEOM) [8, 10]. In case of a stationary stochastic field and product initial states, the expansion of the homogeneous superoperator in cumulants terminates after the first two terms and allows exact solutions $[5,11,12]$. In case of pure dephasing of an atom, when the stochastic field doesn't induce spontaneous transitions between energy levels, the solution could also be obtained analytically $[13,14]$.

We investigate dependence of the non-Markovianity degree of the atom dynamics on the parameters of the stochastic field, the three-level atom parameters, and the three-level atom type. The non-Markovianity degree is evaluated by the "trace distance" measure introduced by Breuer and co-authors [4]. For its calculation, we utilize maximization over randomly selected initial states from the three-level atom state space.

We show that on the interval of evolution from the product initial state, before the measurement, the system dynamics is Markovian and the master equation for the atom density matrix has Lindblad form. After the measurement, the dynamics is non-Markovian for a certain period of time, but only in the case when by the time of measurement, the coherences of the atom are not destructed by the interaction with the stochastic field.

Also, we show that the measurement induces correlations between the previously independent stochastic processes and makes the processes non-stationary.

\section{References}

1. H.-P. Breuer, F. Petruccione, The Theory of Open Quantum Systems (Oxford University Press, 2007)

2. Á. Rivas, S.F. Huelga, M.B. Plenio, Rep. Prog. Phys. 77, 094001 (2014)

3. I. de Vega, D. Alonso, Rev. Mod. Phys. 89, 015001 (2017)

4. E.M. Laine, J. Piilo, H.-P. Breuer, Phys. Rev. A 81, 062115 (2010)

5. V.A. Mikhailov, N.V. Troshkin, Computer Optics 40, 5 (2016)

6. V.G. Morozov, S. Mathey, G. Röpke, Phys. Rev. A 85, 022101 (2012)

7. R. Kubo, J. Math. Phys. 4, 2 (1963)

8. Y. Tanimura, J. Phys. Soc. Jpn. 75, 082001 (2006)

9. M. Ban, S. Kitajima, F. Shibata, Phys. Rev. A 82, 022111 (2010)

10. M. Sarovar, M.D. Grace, Phys. Rev. Lett. 109, 130401 (2012)

11. N.G. van Kampen, Physica 74, 2 (1974)

12. S. Kitajima, M. Ban, F. Shibata, J. Phys. A: Math. Theor. 50, 125303 (2017)

13. M. Ban, S. Kitajima, F. Shibata, Phys. Rev. A 87, 052137 (2013)

14. M. Ban, S. Kitajima, F. Shibata, Phys. Rev. A 87, 022115 (2013) 